\title{
Thermal Decomposition Kinetics Analysis of Citronellol- $\beta$-D-glucopyranoside
}

\author{
Xie Wancui ${ }^{1}$, a , Yin Chao ${ }^{1}$, Yang Xihong $^{2}$, Lei Sheng ${ }^{3}$, \\ Zhang ling ${ }^{3}$, Zhao $\mathrm{Wei}^{3}$ and Zhang Tiandong ${ }^{3}$ \\ ${ }^{1}$ College of Chemical Engineering, Qingdao University of Science \& Technology, Qingdao, China \\ ${ }^{2}$ College of Chemistry and Molecular Engineering, Qingdao University of Science \& Technology, \\ Qingdao, China \\ ${ }^{3}$ Technology Center of China Tobacco Yunnan Industrial Co.Ltd. Kunming, China \\ axiewancui@163.com
}

Keywords: citronellol- $\beta$-D-glucopyranoside (CGLY); TGA/SDTG; DSC; thermal behavior; thermal decomposition kinetics

Abstract. DTA/SDTG and DSC techniques were used to study the thermal behaviors of citronellol- $\beta$-D-glucopyranoside (CGLY). The heating rate was $5,7.5,10$, and $12.5 \mathrm{~K} \cdot \mathrm{min}^{-1}$, the samples were heated from $30^{\circ} \mathrm{C}$ to $500^{\circ} \mathrm{C}$. Methods of Friedman and Flynn-Wall-Ozawa were applied to calculate the parameters of thermal degradation, the parameters were substituted to the methods of Coats-Redfern and Phadnis-Deshpande to estimated degradation mechanism. The TG-DTG curves showed that with the increased heating rate, the mass of starting loss $\mathrm{T} 0$, the biggest rate of mass loss $\mathrm{Tp}$ and final mass loss $\mathrm{T}_{\mathrm{f}}$ met the functions respectively: $\mathrm{T}_{0}=3.8825 \mathrm{~B}+194.97, \mathrm{~T}=2.2825 \mathrm{~B}+281.47$, $\mathrm{Tf}=5.5825 \mathrm{~B}+305.72$. The DSC curve showed that melting and decomposition proceeded at sametime. By Friedman and Flynn-Wall-Ozawa, thermal degradation of the apparent activation energy Ea are obtained, which are $133.15 \mathrm{KJ} / \mathrm{mol}, 126.62 \mathrm{KJ} / \mathrm{mol}$ respectively, and the correlation was related.

\section{Introduction}

Citronellol, 3,7-dimethyl-6-octen-1-ol, is an important fragrant raw materials with sweet floral scent, similar to the smell of roses ${ }^{[1]}$. It often used as an edible flavor for the fragrance of roses, citrus aromas and many citronellol esters and also used as perfuming substance in soft drinks, baked goods, chewing gum, jelly and pudding ${ }^{[2]}$. Citronellol- $\beta$-D-glucopyranoside (CGLY) is a precursor of citronellol. It can be hydrolyzed by glucosidase to release flavor compounds in tea, flowers and other plants, It is an important source of aroma ${ }^{[3,4]}$.

DTA/SDTG and DSC were widely used in the study of thermal stability and degradation. It has gradually become an important means of studying the nature of drugs and natural substances. For example, DTA/SDTG, DSC and on-line Py-GC-MS were uesd to study the thermal degradation behavior of leaf alcohol glycosides ${ }^{[5]}$. The temperature range of the main weight loss of leaf alcohol glycosides and the maximum temperature of release amount of leaf alcohol were obtained. TG-DTG method was used to study the thermal degradation process of salvianolic acid $\mathrm{B}$, the temperature range of the weight loss of salvianolic acid B and the activation energy of the phase were obtained ${ }^{[6]}$.

In this paper, TGA / SDTA and DSC were used to study the thermal degradation behavior of natural identical CGLY under non-isothermal conditions. The thermal degradation kinetics equation was calculated and the thermal degradation mechanism function was ontained. So as to provide important basic data for the study of the thermal properties of flavor precursors and their thermal degradation mechanism.

\section{Experimental}

Materials. Analytically pure citronellol (purity $\geq 92 \%$ ) was purchased from Sigma Company (Shanghai). Sodium acetate, acetic anhydride, bromine, red phosphorus, trifluoroacetic acid, 
methylene chloride, silver carbonate, sodium hydroxide, ammonia, and other reagents were analytically pure.

Synthesis of CGLY. Figure 1 shows the synthesis of CGLY by modified Koenigs-Knorr reaction ${ }^{[7]}$ with $\mathrm{Ag}_{2} \mathrm{CO}_{3}-\mathrm{SiO}_{2}$ as the catalyst. As shown, a $\mathrm{SN}_{2}$ nucleophilic substitution reaction occurred.

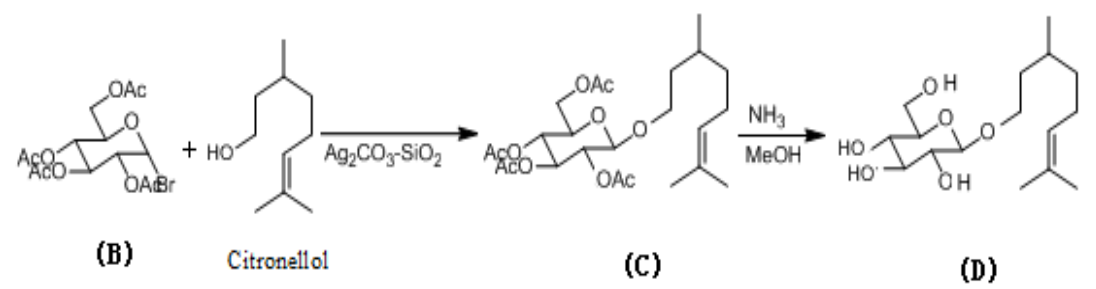

Fig 1. Synthesis scheme of Citronellol- $\beta$-D-glucopyranoside

A solution containing $100 \mathrm{~mL}$ methylene chloride, $10 \mathrm{mmoL}$ (B), and $12 \mathrm{mmoL}$ of citronellol was stirred for $10 \mathrm{~min}$ at room temperature. The newly prepared $\mathrm{AgCO}_{3}-\mathrm{SiO}_{2}$ catalyst was added at $40{ }^{\circ} \mathrm{C}$ reflux after $4 \mathrm{~h}$, cooled to room temperature, filtered, and vacuum-steamed to obtain a pale yellow syrup, i.e., the Citronellol- $\beta$-D-tetraacetyl glycoside (C) products. The solution of compound $\mathrm{C}$ in $\mathrm{MeOH}(100 \mathrm{~mL})$ was bubbled with $\mathrm{NH} 3$ gas at $0{ }^{\circ} \mathrm{C}$ for $1 \mathrm{~h}$. The mixture was stirred for $18 \mathrm{~h}$ at room temperature.

Instruments and conditions. The apparatus used was an Agilent 1200 LC, Agilent Technology; ODS-AQ C18 250×20mm Column, ODS-AQ C18 250×4.6 mm Column, YMC Co.; TGA/SDTA851e TG/DSC, Switzerland Mettlerto ledo company.

About 5mg of the CGLY samples were taken in aluminum pan and subjected to the DSC (TGA /SDTA 851e) at five different heating rates (5、7.5、10、12.5 K/min). High-purity nitrogen was used as a purge gas at a flow rate of $30 \mathrm{~mL} / \mathrm{min}$. The temperature range covered in DSC was from $30 \square$ to $500 \square$.

The theory of solid thermal degradation. In general, the thermal degradation process of a solid compound can be expressed as : $A_{\text {solid }} \rightarrow B_{\text {solid }}+C_{\text {gas }}$, where represents the reactants, $B_{\text {solid }}$ and $C_{\text {gas }}$ are generated by the thermal degradation. The degree of the thermal degradation of $A_{\text {solid }}$ (conversion rate) can be expressed as

$$
X=\frac{W_{0}-W_{t}}{W_{0}-W_{f}}
$$

where $\mathrm{X}$ is the conversion rate of the reactants, $\mathrm{W}_{0}$ is the initial quality of the sample, $\mathrm{W}_{\mathrm{t}}$ is the quality of the sample in time $\mathrm{t}$, and $\mathrm{W}_{\mathrm{f}}$ is the final quality of the sample.

The conversion rate changes with the increasing of reaction time, and we can get the following formula by differentiating it

$$
\frac{\mathrm{dX}}{\mathrm{dt}}=\mathrm{kF}(\mathrm{X})
$$

where $\frac{\mathrm{dX}}{\mathrm{dt}}$ is the degradation rate of the reactants, $k$ is the degradation rate constant, and $\mathrm{F}(\mathrm{X})$ is the function based on the degradation principle.

Suppose that k satisfies the Arrhenius equation, the degradation rate constant can be expressed as

$$
\mathrm{k}=\mathrm{A} \exp \left(\frac{-\mathrm{Ea}}{\mathrm{RT}}\right)
$$

where A is pre-exponential factor(s-1), Ea is activation energy $(\mathrm{J} / \mathrm{mol}), \mathrm{R}$ is molar gas constant (

$8.314 \mathrm{~J} / \mathrm{mol} / \mathrm{K}$ ), and $\mathrm{T}$ is Kelvin temperature ( K ) . Substituting (3)into(2), we have

$$
\frac{\mathrm{dX}}{\mathrm{dt}}=\mathrm{A} \exp \left(\frac{-\mathrm{Ea}}{\mathrm{RT}}\right) \mathrm{f}(\mathrm{X})
$$

In thermo-gravimetric analysis, if the temperature $\mathrm{T}(\mathrm{T}=\mathrm{T} 0+\mathrm{Bt})$ rises linearly according to some given rate $\mathrm{B}$, then the change of the degradation degree of the reactions can be characterized as a formula that is related to the temperature. By substituting $\mathrm{dt}=\mathrm{dT} / \mathrm{B}$ into (4), we can get 
$\frac{\mathrm{dX}}{\mathrm{f}(\mathrm{X})}=\frac{\mathrm{A}}{\mathrm{B}} \exp \left(\frac{-\mathrm{Ea}}{\mathrm{RT}}\right) \mathrm{dT}$

and Equation (5) are the fundamental formulas in thermal degradation computations.

Friedman method. The Friedman method is a derivative method ${ }^{[8]}$ based on formula (4), and its main formula is

$$
\ln \left(\mathrm{B} \frac{\mathrm{dX}}{\mathrm{dT}}\right)=\ln [\mathrm{Af}(\mathrm{X})]-\frac{\mathrm{Ea}}{\mathrm{RT}}
$$

According to (6), if we draw the curve of $\ln \left(\mathrm{B} \frac{\mathrm{dX}}{\mathrm{dT}}\right)$ against $1 / \mathrm{T}$, then the Ea under different conversion rate can be obtained.

Flynn-Wall-Ozawa method. Taking the integral of the Equation (5) ${ }^{[9-10]}$, and its main formula is

$$
\lg B=\lg \frac{\mathrm{AEa}}{\mathrm{g}(\mathrm{X}) \mathrm{R}}-2.315-0.4567 \frac{\mathrm{Ea}}{\mathrm{RT}}
$$

Therefore, under the conditon that the conversion rate is fixed to be a sequence of special values, by drawing the curve of $\log \mathrm{B}$ against $1 / \mathrm{T}$, the Ea under different conversion rate can be obtained.

\section{Results and discussion}

Thermal gravimetry analysis. Fig.2 was the TG-DTG curves of CGLY from $30 \square$ to $500 \square$ with the heating rate of $5,7.5,10$ and $12.5 \mathrm{~K} \cdot \mathrm{min}-1$.

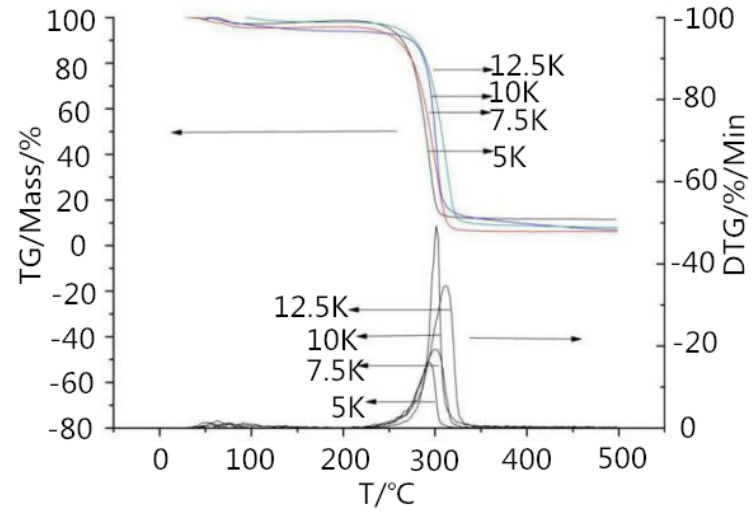

Fig. 2 The TG-DTG curve of the decomposition of CGLY

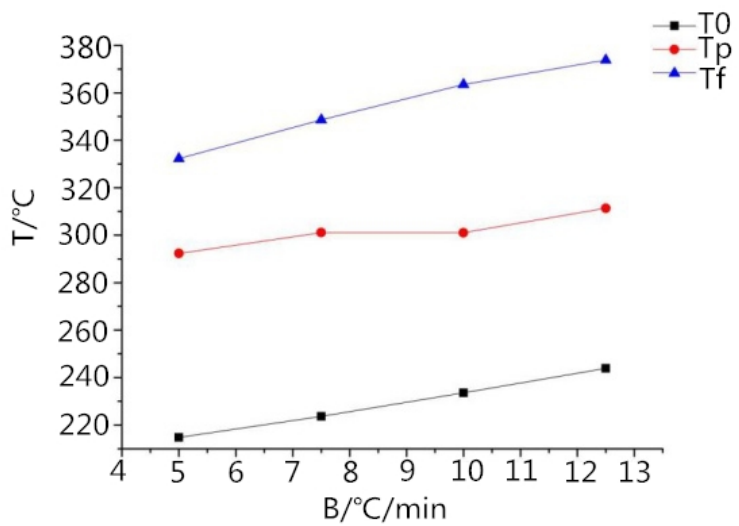

Fig. 3 The relationship of heating rate $B$ and degradation temperature

The result showsthe thermal degradation process of CGLY was the only step of the thermal degradation process.. The step mass loss was due to the degradation of the CGLY, which indicated the only step of thermal degradation process.

The influence of heating rate on the thermal degradation temperature. Initial temperature of mass reduction $T_{0}$ and final temperature of mass reduction $T_{\mathrm{f}}$ were obtained by drawing tangent to $\mathrm{TG}$ curve. And temperature of mass reduction at the maximum rate $\mathrm{T}_{\mathrm{p}}$ was obtained from DTG curve.

Fig. 3 shows the relationship between thermal degradation temperature and heating rate. It can be obtained from Fig. 3 that $\mathrm{T}_{0}=3.8825 \mathrm{~B}+194.97$ with a correlation coefficient of 0.99877 ; $\mathrm{T}_{\mathrm{p}}=2.2825 \mathrm{~B}+281.47$ witha correlation coefficient of $0.89556 ; \mathrm{T}_{\mathrm{f}}=5.5825 \mathrm{~B}+305.72$ with a correlation coefficient of 0.99023 . Thermal degradation temperature is mainly affected by the heating rate.

Effect of heating rate $B$ on degradation degree. Table 1 shows the relationship between the heating rate $B$ and the degradation degree $X . X_{p}$ and $X_{f}$ are respectively the conversion rates of CGLY at $T_{p}$ and $T_{f}$.

\begin{tabular}{|c|c|c|c|c|}
\hline $\mathrm{B} / \mathrm{K} \cdot \min ^{-1}$ & 5 & 7.5 & 10 & 12.5 \\
\hline $\mathrm{X}_{\mathrm{p}}$ & 58.9 & 66.1 & 56.3 & 61.8 \\
\hline $\mathrm{X}_{\mathrm{f}}$ & 88.9 & 93.7 & 89.9 & 90.9 \\
\hline
\end{tabular}

Table 1. Heating rate and its influence on degradation rate 
The degradation rate did not change much at different heating rates. The average degradation rate of $\mathrm{X}_{\mathrm{p}}$ is $60.78 \%$ at temperature $\mathrm{T}_{\mathrm{p}}$ and $90.9 \%$ at temperature $\mathrm{T}_{\mathrm{f}}$. Degradation degree is mainly affected by thermal degradation temperature.

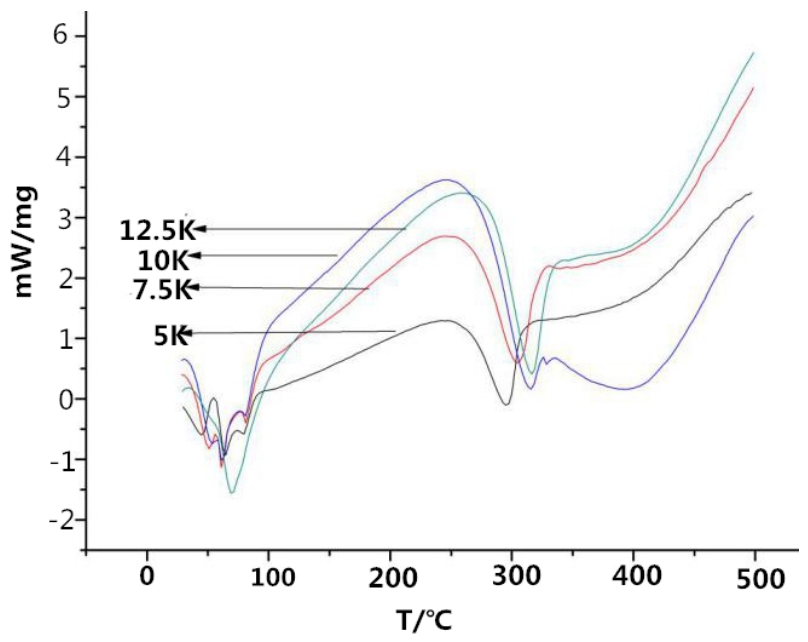

Fig. 4 The DSC curve of decomposition of citronellol glycoside

Fig.4 shows the DSC curves for the CGLY. A supreme endothermic peak is in the range of 245 $\sim 335 \square$, which agrees the main degradation interval in TG and DTG curves. Except for the endothermic peak caused by expulsion of water, there is only one peak which indicates CGLY did not undergo phase transition during the thermal degradation process. The DSC curve showed that the process of melting and decomposition proceeded at the same time.

Ea of CGLY in decomposition. Fig.5 (a) shows that using Friedman Method, the plot of lnB vs.1/T produced traight lines, the slope of which indicated the activation energy $\left(\mathrm{Ea}_{1}\right)$ of CGLY during decomposition at different T. Fig. 5(b) shows that using Flynn-Wall-Ozawa Method, the plot of $\operatorname{lgB}$ vs.1/T gives traight lines, the slope of which indicate the activation energy $\left(\mathrm{Ea}_{2}\right)$ of CGLY in decomposition at different $\mathrm{T}$.

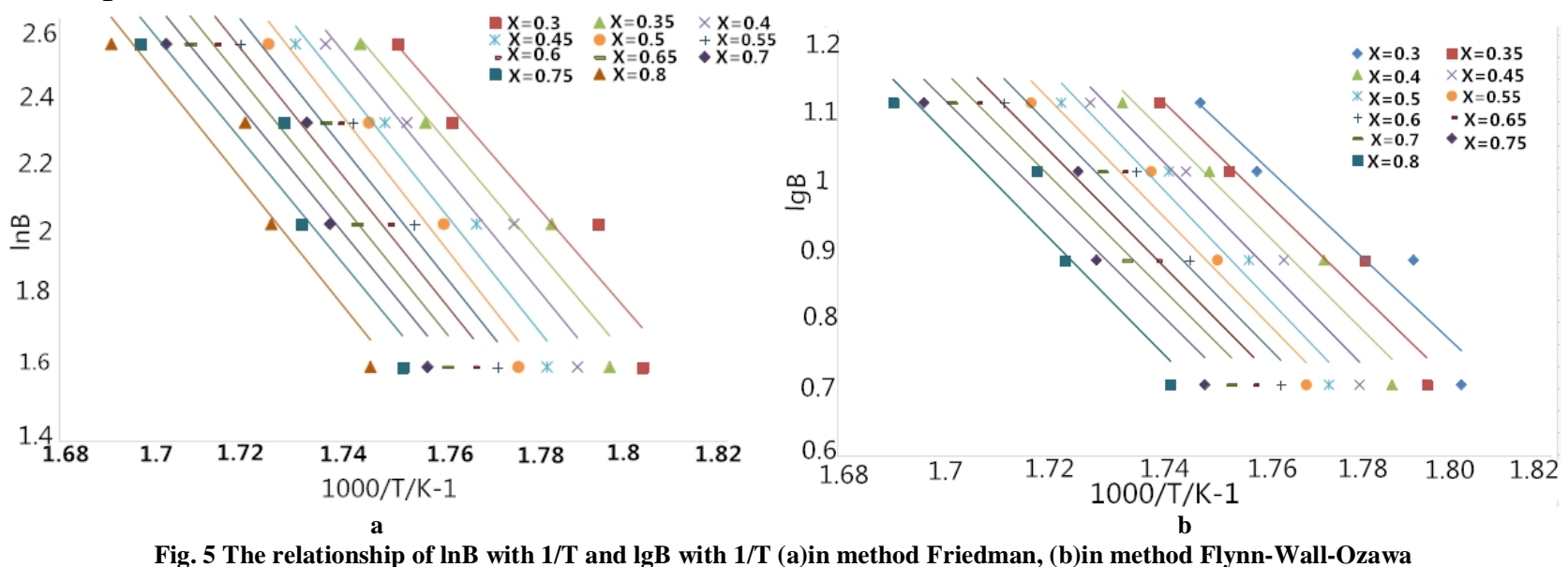

Fig.6 presents the dependence of activation energy of CGLY in decomposition based on Friedman method and Flynn-Wall-Ozawa method. 


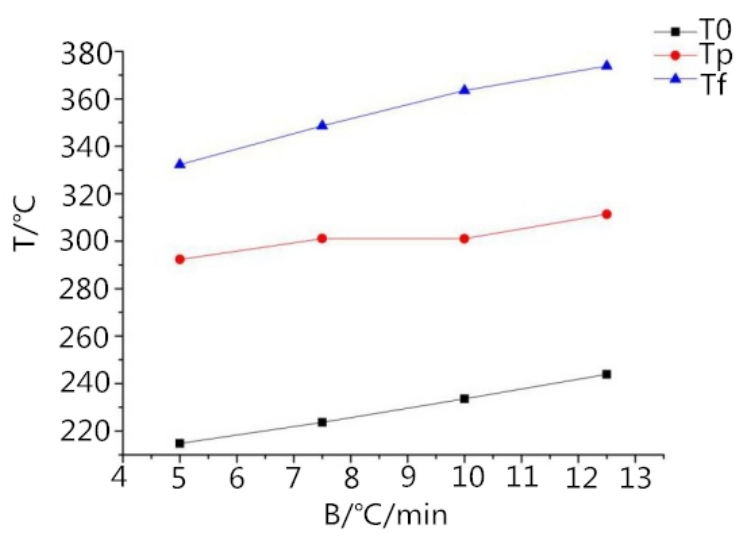

Fig. 6 Ea of thermal degradation of CGLY by two methods

From Fig.6 it shows that activation energies calculated by using different methods were comparable and the average activation energies are $133.15 \mathrm{~kJ} / \mathrm{mol} 、 126.62 \mathrm{~kJ} / \mathrm{mol}$ which implied that the kinetic parameters obtained are reasonable.

\section{Conclusions}

The thermal degradation process and thermal decomposition kinetics of CGLY were studied by DTA / SDTG and DSC. The experimental results are shown as following:

(1) TG-DTG curves showed that the thermal degradation temperature was mainly affected by the heating rate, and it was gradually increasing with the enhancement of heating rate. The DSC curve showed that the degradation temperature was in the range from $245 \square$ to $335 \square$, and the process of melting and decomposition proceeded at the same time.

(2) The average activation energies are respectively $133.15 \mathrm{~kJ} / \mathrm{mol} 、 126.62 \mathrm{~kJ} / \mathrm{mol}$ obtained by Friedman method and Flynn-Wall-Ozawa method, and the correlation was related, which indicated that the kinetic parameters obtained were reasonable.

The results showed that CGLY which is the precursor of citronellol aroma, improved the thermal stability of citronellol . It was important for the application of CGLY in high temperature heating food or cigarette flavoring.

\section{Acknowledgments}

The authors acknowledge the financial support given by the Chinese National Natural Science Foundation (No. 21275035), the science and technology projects of Yunnan ZhongYan Industrial Co., LTD Technology Center (No.HYHH2013HX03), and the science and technology projects of Yunnan Zhong Yan Industrial Co., LTD of (No.2013CP02).

\section{Literature References}

[1] Rui-hong ZHU, Qun-cheng XUE, Zhong-cheng LI. Handbook of Synthetic Food Flavor [M] Shandong: Jinan Light Industry Research Institute, 1991.49-50.(in Chinese)

[2] Hui-juan KONG. Extraction, separation and characterization of six glycosidic bond aromas components in fruits [D]: Zhejiang University, 2016.(in Chinese)

[3] R. Noguerol-Pato, C. González-Barreiro, B. Cancho-Grande, J. L. Santiago, M. C. Martínez, J. Simal-Gándara. Aroma potential of Brancellao grapes from different cluster positions [J]. Food Chemistry, 2012, 132(1): 112-124. 
[4] W. C. Xie, X. H. Yang, C. H. Zhang, et al. Investigation on pyrolysis behavior and decomposition compounds of three synthesized glycosidically bond flavor precursors. Thermochimica Acta, 2012, 532: 2-9

[5] W.C. Xie, Z.C. Tan, X.H. Gu, et al. Thermal decomposition of two synthetic glycosides by TG, DSC and Simultaneous Py-GC-MS analysis[J]. Journal of Thermal Analysis and Calorimetry. 2007, 87(2): 505-510.

[6] Shao-Xu WANG, Zhi-Cheng TAN.Studies on Thermal Stability and Thermal Decomposition Kinetics of Salvianolic Acid B [J]. Acta Chimica Sinica, 2012, 70 (2): 212-216. (in Chinese)

[7] Wan-cui Xie, Jian Tang, Xiao-hong Gu, et al. Thermal decomposition study of menthyl-glycoside by TGA/SDTA, DSC and simultaneous Py-GC-MS analysis[J]. J. Anal. Appl. Pyrolysis. 2007, 78: $180-184$.

[8] Popescu C.Intergral method to analyze the kinetics of heterogeneous reactions non-isothermal conditions a variant on the Ozawae Flynne Wall method[J]. Thermochimica Acta, 1996, 285(1): 9 23.

[9] Dowdy DR. Meaningful activation energies for complex systemsi. The application of Ozawa-Flynn-Wall method to multiple reactions[J]. J. Therm Anal, 1987, 32 : 137-139.

[10] BY Liu, XJ Zhao, XH Wang, et al. Thermal degradation Kinetics of poly(propylene carbonate)obtained from the copolymerization of carbon dioxide and propylene oxide[J]. J Appl Polym Sci, 2003, 90: 46 53. 